\title{
Electron-Positron Pairs Related to Alfven Waves on the Magnetar Surface
}

\author{
Abdul Kader, Kalpana Duorah \\ Department of Physics, Gauhati University, Guwahati, Assam, India \\ Email address: \\ kader.abdul781@gmail.com (A. Kader), khowang56@yahoo.co.in (K. Duorah) \\ To cite this article: \\ Abdul Kader, Kalpana Duorah. Electron-Positron Pairs Related to Alfven Waves on the Magnetar Surface. International Journal of \\ Astrophysics and Space Science. Vol. 3, No. 3, 2015, pp. 25-29. doi: 10.11648/j.ijass.20150303.11
}

\begin{abstract}
The phenomena of electron-positron pairs production through the polar cap region of a magnetar is studied. Assuming the distances of electron-positron pair regions from the magnetar centre to be the height scales of magnetic deformed regions and hence determine by adopting the Alfven waves propagation. The maximum potential drop on the magnetar surface and the potential differences in the confined regions are obtained by using Lorentz factor. The attempt is made to find out the curvature photon energy through the polar cap region of magnetar that is greater than critical energy and verify the conversion into electron-positron pairs via synchrotron radiation with their characteristic photon energy. Further, an estimate is made to flow the charged particles per second through the polar cap region.
\end{abstract}

Keywords: Electron-Positron Pairs, Polar Cap Region, Magnetar, Alfven Waves, Critical Energy, Lorentz Factor

\section{Introduction}

Magnetars are neutron stars with high magnetic field strengths, $B \gtrsim 10^{14} G$. Magnetars are believed to be two classes: Soft Gamma-ray Repeaters (SGRs) and Anomalous X-ray Pulsars (AXPs). Thompson et al. [1] suggested that the highly twisted internal magnetic fields $\sim\left(10^{14}-10^{16}\right) G$ stress the crust and Thompson \& Duncan [2] predicted that the crustal dislocations or disturbances (e.g. shear waves) excite Alfven waves in the magnetar magnetosphere. Blaes et al. [3] argued that the neutron star quake will excite the oscillation of the magnetic field frozen into the star's crust, and the Alfven waves carrying the released energy into the magnetosphere. In the fireball model Thompson and Duncan [2] argued that the Alfven waves will provide the energy to produce a photon of electron-positron pairs. Motions of the relativistic electrons are restricted along the strong magnetic field lines and they radiate curvature photons. The photons can escape freely from the surface but electrons and positrons annihilate, then the trapped fireball is radiated away. The dissipation of wave energy will accelerate the electrons and positrons. In the magnetar model, it can be thought that the Alfven wave is excited in the magnetic field lines anchored on the deformed position. Thompson and Duncan [2] proposed that the minimum Alfven excitation radius will not be much greater than that of the neutron star in the case where the patch of the crust is taken to be fractured.
Cumming et al. [4] suggested that the length scale of disturbance region may not be of the order of the size of the region but rather its local pressure scale height $l \sim H=P / \rho g$, where $\mathrm{g}$ is the acceleration due to gravity, $P$ is the local pressure and $\rho$ is the density of the neutron star. If the cracking location is close to the magnetic pole, the released energy can be carried out by the Alfven waves into the outer magnetosphere.

It is predicted that the rotating strongly magnetized neutron stars generate huge potential difference between the centre and outer edge of polar cap region. The associated electric fields are able to accelerate charged particles. In the magnetosphere, these particles can flow only along the field lines and they escape through the light cylinder, beyond which the field lines become open. This out flow of charged particles from the co-rotating magnetosphere causes a gap near the surface where $E . B \neq 0$ becomes unstable when curvature photons produce an avalanche of electron-positron pairs [5]. Although SGRs and AXPs are slowly rotating huge magnetic field neutron stars, they can accelerate particles and emit photons [6].

Charged particles are expected to be accelerated either in the polar cap region near the neutron star surface $[5,7,8]$ or above the null charge surface [9]. The accelerated primary particles are believed to be radiated either through curvature radiation or inverse Compton Scattering, while the resultant gamma-rays produce electron-positron pairs either through one photon $\left(\gamma(B) \rightarrow e^{+} e^{-}(\mathrm{B})\right)$ or two photons $\left(\gamma \gamma \rightarrow e^{+} e^{-}\right)$ 
processes. In the polar cap region near the magnetar surface, the secondary electron-positron pairs radiate through synchrotron radiation and inverse Compton Scattering that leading to a photon-pair cascade $[10,11]$. In this paper we will concentrate on a class of models that the charged particles are supplied from the magnetar surface along open field lines. It is predicted that these charged particles are limited either by binding energy or cohesive energy and therefore can reach the Goldreich-Julian rate close to the surface called Space Charge Limited Flow [12, 7].

Chi et al. [13] proposed that curvature photons would be converted into secondary electron-positron pairs in the local magnetic field when the curvature energy $E_{\text {cur }}>$ $E_{\text {crit }}$ (critical energy) and these $e^{ \pm}$pairs would be lost their energies via synchrotron radiation. A self-consistent mechanism was developed by Zhang and Cheng [14] to interpret high energy radiation from spin-powered pulsars. The model proposed by Cheng and Zhang [15] that the relativistic charged particles radiate from outer gap through the synchro-curvature radiation mechanism rather than the synchrotron and curvature mechanisms. In this model, generally the producing non-thermal photons from the primary electron-positron pairs will move along the curved magnetic field lines in the outer gap. It has been developed by Ruderman and Sutherland [5] that the electrons pulled out from the neutron star surface are accelerated and emitted curvature radiation(photons). These emitted photons produce electron-positron pairs. Again these secondary $e^{ \pm}$pairs can produce curvature radiation, that will produce new $e^{ \pm}$pairs, and so on.

The key point of the paper is to find out the radiation of curvature photons through the polar cap region of magnetar and their conversion into electron-positron pairs via synchrotron radiation. We determine the height scales of magnetic deformed regions by considering the Alfven waves travel at the same speed of light and each of the deformed regions is assumed to be spherical surface to observe the electron-positron pair production. Adopting the electronpositron out flowing regions, our model is confined within the spherical surfaces of radii $R \approx 10^{6} \mathrm{~cm}$ and $r \approx 2.5 \times$ $10^{6} \mathrm{~cm}$. The origin of the abrupt disturbances relevant to the bursts is beyond of our model, but the subsequent propagation and ejection of Alfven waves to the exterior are examined. In the section 2 we discuss the Alfven waves propagation through the polar cap region near the magnetar surface. In section 3 we attempt to find out the curvature photons that converted into secondary $e^{ \pm}$pairs and lose their energies via synchrotron radiation with photon energy within our confined region. The results and discussions are presented in section 4 and conclusions are made in section 5 .

\section{Alfven Waves Propagation on the Magnetar Surface Near Polar Cap}

Thompson and Duncan [2] proposed that a magnetic disturbance is occurred on the magnetar crust due to the stresses of interior strong magnetic field. The displacement of the magnetic field lines on the crust would be a result of the emission of Alfven waves in the magnetosphere [2]. The pattern of Alfven wave excitation in the magnetosphere depends upon the exterior field geometry in the case where a patch of the crust fractures and shifts with a small horizontal displacement that the magnetic field decays over with Alfven velocity $v_{A} \sim \frac{B_{S}}{\sqrt{4 \pi \rho}}$ [2] where $B_{S}$ is the surface magnetic field and $\rho$ is the density of the magnetar. In the magnetosphere of magnetar, Alfven waves travel with velocity very close to the speed of light, $v_{A} \sim c \approx 3 \times 10^{10} \mathrm{~cm} \mathrm{~s}^{-1}$ [2]. Cumming et al. [4] suggested the local pressure scale height will be $r \sim H=$ $P / \rho g$. If the local pressure $P$ is assumed to be a magnetic pressure $\simeq B_{S}^{2} / 8 \pi$ then we can find out the maximum height scale of magnetic disturbance region

$r \approx \frac{v_{A}^{2}}{2 g} \approx 2.5 \times 10^{6}\left(\frac{v_{A}}{3 \times 10^{10} c m s^{-1}}\right)^{2}\left(\frac{g}{1.7 \times 10^{14} c m s^{-2}}\right)^{-1} \mathrm{~cm}$,

from the centre of magnetar. Here, $g$ is the acceleration due to gravity $\approx \frac{G M}{R^{2}}$, where $\mathrm{G}$ is the Universal Gravitational constant $\approx 6.67 \times 10^{-8} \mathrm{erg} \mathrm{cm}^{2} \mathrm{~g}^{-2}, \mathrm{M} \sim$ mass of the magnetar $\approx 1.4 M_{\odot}$ and $\mathrm{R}=10^{6} \mathrm{~cm}$, radius of the magnetar.Thompson \& Duncan [2] proposed that the minimum Alfven excitation radius will not be much greater than that of the neutron star in the case where the patch of the crust is taken to be fractured. In our model, we consider the height scales of disturbance regions range from $R \approx 10^{6} \mathrm{~cm}$ to $r \approx 2.5 \times 10^{6} \mathrm{~cm}$ from the magnetar centre. It has been thought that the transport of magnetic field strength on the magnetar surface produces Alfven waves at a local distance $r$ from the magnetar centre [16]

$$
B_{r} \approx B_{s}\left(\frac{R}{r}\right)^{2},
$$

where $B_{S} \sim 6 \times 10^{14} G$, dipole magnetic field strength of a magnetar [2]. It has been predicted that magnetar could be considered as an oblique rotator with a strong magnetic field strength $B_{S}$ and angular spin $\Omega$ that gives rise Goldreich and Julian [17] charge density $\rho \simeq \Omega . B_{S} / 2 \pi c$ in the corotating magnetosphere. The open field lines intersect the null charge surface $\Omega . B=0$ within the light cylinder just above the last closed field line and the charge density changes sign at this surface. The outer gaps arise as the charges escaping through the light cylinder along open field lines above the null charge surface that cannot be replenished from the magnetar surface [9].A model was proposed by Ruderman and Sutherland [5] of pulsar emission based on the formation of vacuum gaps near the polar caps. In this model the work function for charged particles on the neutron star surface is assumed to be high enough to prevent them from being stripped from the surface and shorting out the potential that is induced. Based on the model proposed by Ruderman and Sutherland [5], we introduce a maximum potential is thereby developed in the polar cap region on the magnetar surface that can be estimated as [18]

$$
V_{\max }=\frac{\Omega^{2} B_{S} R^{3}}{2 c^{2}}
$$

with the rotation frequency $\Omega=2 \pi / p$, where $p$ is the 
rotational period, $\mathrm{c}$ is the light speed $3 \times 10^{10} \mathrm{cms}^{-1}$. The expression $B_{S} R^{3}$ represents the magnetic dipole moment of the magnetar. For magnetar we assume the rotational period $(5-8) s[19]$ and hence the maximum potential on the magnetar surface is obtained as $V_{\max } \approx 8 \times 10^{13}$ volt. Near the magnetar surface the potential difference can be calculated by using the space charge limited flow (SCLF) model proposed by Muslimov and Tsygan [20]. In applying the space time solution to the electrodynamics of the Polar Cap SCLF model, the part of the induced electric field will be left unshielded, and a potential difference at a distance $r$ $(\mathrm{r}>R)$ from the stellar centre is obtained through the relation [21]

$$
V_{r} \approx V_{\max } \kappa_{g}\left[1-\left(\frac{R}{r}\right)^{3}\right]
$$

for $\mathrm{r}>R$. Here $\kappa_{g}=\frac{I}{M R^{2}} \cdot \frac{R_{g}}{R}$ known as Relativistic parameter [20] with $R_{g}=\frac{2 G M}{C^{2}}$ is called Schwarzschild radius, $I=$ moment of inertia of magnetar $\sim 10^{45} \mathrm{gcm}^{2}$. A remarkable result is obtained by Muslimov and Tsygan [20] for $k_{g} \simeq$ $0.15 I_{45}$. Our model is confined within the spherical surfaces of radii $R \approx 10^{6} \mathrm{~cm}$ and $r \approx 2.5 \times 10^{6} \mathrm{~cm}$ and within this limitation the local pressure is considered to be a magnetic pressure.

\section{Electron-Positron Pairs in the Magnetosphere}

A self-consistent mechanism was developed by Zhang and Cheng [14] to interpret high energy radiation from spinpowered pulsars. It was proposed by Arons [22] that in the polar cap region the electrons are freely emitted from the neutron star surface and accelerated towards the magnetosphere. Accelerated primary particles can radiate through curvature radiation as well as inverse Compton photons that can produce electron-positron pairs in the polar cap region in the presence of strong magnetic field [9]. In the polar cap region, the secondary $e^{ \pm}$pairs radiate through synchrotron radiation, while inverse Compton photons leading to a photon pair cascade $[10,18]$. It is noted that some of the positrons change their direction and accelerate downward toward the star, while the electrons continue stream outward. In this paper we examine the secondary $e^{ \pm}$ pairs will lose their energies via synchrotron radiation with photon energy at a distance $r$ (different distances are considered) from the stellar centre. The fireball model [2] introduced that the Alfven waves will carry out the energy and momentum to produce a photon electron-positron fireball. Ruderman and Sutherland [5] model introduced the Lorentz factor of primary electrons $\sim \gamma \simeq e \Delta V / m_{e} c^{2}$, here $\mathrm{e}$ is the electronic charge, $\Delta V$ is the typical potential drop across the open field lines, and $m_{e}$ is the rest mass of the electron. The pair production mechanism is a synchrotron photon cascade in a strong magnetic field. Halpern and Ruderman [23] proposed that each primary electron-positron from an outer gap near the polar cap will contain energy. In our model, each primary electron-positron from the polar cap will have an energy [23]

$$
E_{p}=\gamma m_{e} c^{2}
$$

It is vital that the electrons are accelerated across the potential drop that able to create energetic photons by curvature radiation, which can produce $e^{-} e^{+}$off the magnetic field.These particles are restricted to move along the strong magnetic field lines and therefore they will radiate curvature photons with a characteristic energy [5]

$$
E_{\text {cur }}=\frac{3}{2} \gamma^{3}\left(\frac{c \Omega}{R}\right)^{\frac{1}{2}} \hbar,
$$

where $\hbar$ is the Planck constant $\sim 1.16 \times 10^{-16} \mathrm{eV}$. We calculate the energy of radiated curvature photons and obtain the maximum value $\sim 10^{11} \mathrm{eV}$ on the magnetar surface and minimum value $\sim 5.4 \times 10^{7} \mathrm{eV}$ near the magnetar surface. These curvature photons cannot escape from the magnetar surface due to the strong magnetic field and they have to be converted into $e^{ \pm}$pairs to emit from the surface [24]. Further, it is predicted that these curvature photons will be converted into secondary electron-positron pairs in the local magnetic field of polar cap region, when their energies satisfy the condition [5]

$$
E_{\text {cur }} \geq \frac{2 m_{e} c^{2}}{15} \frac{B_{\text {crit }}}{B_{S}} \simeq 10^{4}\left(\frac{B_{S}}{10^{14} G}\right)^{-1} \mathrm{eV} \equiv E_{\text {crit }},
$$

where $E_{\text {crit }}$ is the critical energy for the critical magnetic field $B_{\text {crit }}=4.4 \times 10^{13} G$ and $B_{s}$ is the local magnetic field on the magnetar surface. The secondary $e^{ \pm}$pairs will emit their energies through the polar cap region via synchrotron radiation with photon energy [13]

$$
E_{\text {syn }}=\frac{3}{2}\left(\frac{E_{c u r}}{2 m_{e} c^{2}}\right)^{2} \hbar\left(\frac{\omega_{B}}{B}\right) B_{r}
$$

For accelerated electrons in the magnetosphere, the synchrotron frequency, $\omega_{B}=\frac{e B}{m_{e} c}$ implies that $\frac{\omega_{B}}{B}=\frac{e}{m_{e} c} \approx$ $1.76 \times 10^{7} \mathrm{rad} \mathrm{s}^{-1} G^{-1}$. On the magnetar surface $B_{r}=B_{S}$ and we observe maximum synchrotron energy $E_{\text {syn }} \approx$ $10^{16} \mathrm{eV}$ implies $E_{\text {syn }} \gg E_{\text {crit }}$ so that a photon-electron cascade will start and develop until this condition fails. The Goldreich and Julian [17] charge density $\rho \simeq-\Omega \cdot \frac{B}{2 \pi c}$ in the polar cap region of a magnetar provides an upper limit of the net primary electron-positrons flow in the magnetosphere. It is expected that the flow of primary particles (electronpositron) through polar cap [17]

$$
\dot{N} \approx \frac{B_{r} \Omega^{2} r^{3}}{2 e c},
$$

gives an upper limit $\dot{N} \sim 4.2 \times 10^{31} s^{-1}$ for the maximum height scale $r \sim 2.5 \times 10^{6} \mathrm{~cm}$ in the confined region and the lower limit $\dot{N} \sim 1.7 \times 10^{31} \mathrm{~s}^{-1}$ on the magnetar surface at $r=R$ and $B_{r}=B_{S}$. Further, the equation (9) is predicted to hold for any surface field distribution with a dipole moment $B_{s} R^{3}$ adjusted to fit the observed magnetars spin-down rate. 


\section{Results and Discussions}

The values of height scales (r) contained in Table 1 are assumed to be the distances of $e^{ \pm}$pair production regions from the magnetar centre and each region is considered to be spherical one. Adopting the height scales, we calculate the magnetic field strengths $\left(B_{r}\right)$ by applying the equation (2), and potential differences by using the equations (3) and (4). The Lorentz factor $(\gamma)$ of electron and positron that depends on the potential difference, the energy of radiated curvature photons $\left(E_{\text {cur }}\right)$ as well as the energy of the synchrotron radiation $\left(E_{s y n}\right)$, and the particles per second $(\dot{N})$ flow through the polar cap for different height scales (r) from the magnetar centre; these are calculated and listed in Table 1.

Table 1. height scales $(r)$ of $e^{ \pm}$pairs production regions from the magnetar centre; the magnetic field strengths ( $\left.B_{r}\right)$, potential difference $\Delta V$, Lorentz factor $\gamma$,Curvature radiated energy $E_{\text {cur }}$, Synchrotron energy $E_{\text {syn }}$, and particles (electron-positrons) flow per second $\dot{N}$.

\begin{tabular}{|c|c|c|c|c|c|c|}
\hline $\begin{array}{l}\text { Height scale } \\
r \text { in }\left(10^{6} \mathrm{~cm}\right)\end{array}$ & $\begin{array}{l}\text { Mag. field } \\
B_{r}\left(10^{14} G\right)\end{array}$ & $\begin{array}{l}\text { Pot.Dif. } \Delta V \\
\left(10^{13} \text { volt }\right)\end{array}$ & $\begin{array}{l}\text { Lorentz } \\
\text { factor } \gamma\end{array}$ & $\begin{array}{l}\text { Cur. Energy } \\
E_{c u r} \text { in eV }\end{array}$ & $\begin{array}{l}\text { Syn. Energy } \\
E_{\text {syn }} \text { in } \mathrm{eV}\end{array}$ & $\begin{array}{l}\text { Particle flow } \\
\dot{N} \text { in }\left(10^{31} s^{-1}\right)\end{array}$ \\
\hline 1.0 & 6 & 8 & $15.6 \times 10^{7}$ & $1.1 \times 10^{11}$ & $2.1 \times 10^{16}$ & 1.7 \\
\hline 1.3 & 3.6 & 0.65 & $1.25 \times 10^{7}$ & $5.44 \times 10^{7}$ & $3.1 \times 10^{9}$ & 2.2 \\
\hline 1.5 & 2.7 & 0.8 & $1.58 \times 10^{7}$ & $1.1 \times 10^{8}$ & $0.95 \times 10^{10}$ & 2.5 \\
\hline 1.8 & 1.85 & 0.9 & $1.9 \times 10^{7}$ & $1.9 \times 10^{8}$ & $1.96 \times 10^{10}$ & 3.0 \\
\hline 2.0 & 1.5 & 1.05 & $2 \times 10^{7}$ & $2.2 \times 10^{8}$ & $2.13 \times 10^{10}$ & 3.4 \\
\hline 2.3 & 1.13 & 1.1 & $2.15 \times 10^{7}$ & $2.76 \times 10^{8}$ & $2.52 \times 10^{10}$ & 3.8 \\
\hline 2.5 & 0.96 & 1.12 & $2.16 \times 10^{7}$ & $2.8 \times 10^{8}$ & $2.20 \times 10^{10}$ & 4.2 \\
\hline
\end{tabular}

In the Table 1, it is noted that the electron-positron pairs flowing out through the polar cap region increases with the height scale. Furthermore, recalling the diagram of the traditional magnetic dipole model of a pulsar, it is predicted that the increase of flow of electron-positron pairs depend on the polar cap radius around the dipole axis in the polar cap region, $r_{p c} \approx r\left(R / R_{L C}\right)^{\frac{1}{2}}$ where $R_{L C}$ is the light cylinder radius $[25,26]$.

We consider the Alfven waves produced in the magnetic deformed regions by displacing the magnetic field lines in a rotating magnetar. The origin of abrupt magnetic disturbances relevant to the burst mechanism is not addressed here, but the subsequent propagation and ejection of Alfven waves to the exterior are discussed. Adopting the suggestion of Cumming et al. [4] for local pressure and acquiring the conception of Thompson \& Duncan [2] for propagation of Alfven waves in the magnetosphere; we calculate the height scales of disturbance regions from the magnetar centre to study the $e^{ \pm}$pair production mechanism.

Below, the figure 1 shows the energy radiated by the curvature photon gradually increases with Lorentz factor while the energy radiated through synchrotron radiation in the initial stage within the range from Log.7.2 to Log.7.4 gradually increases and after then tremendously increases.

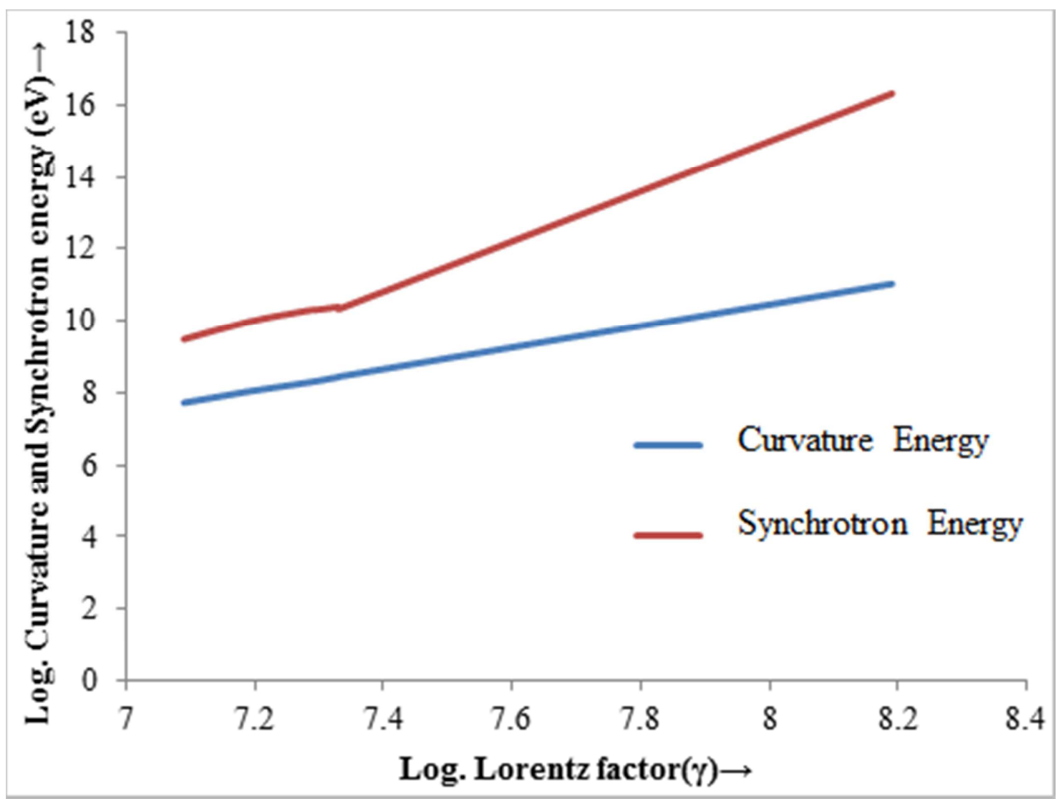

Figure 1. Log. Lorentz factor $(\gamma)$ against Log. Curvature and Synchrotron energy in eV for magnetars are plotted. Blue solid line represents the energy radiated by curvature photons while red solid line represents the energy radiated through synchrotron radiation.

\section{Conclusions}

In this paper we have studied and observed the behavior of $e^{ \pm}$pairs that created by curvature photons in the polar cap region of magnetar by developing different models. The concerned models: magnetar model, polar cap model, space charge limited flow model, and polar gap model. Also the other important phenomena like as Alfven waves 
propagation and transportation in the magnetosphere of magnetar is discussed here.

In our model all the calculations are obtained by assuming the dipole magnetic field strength $B_{S}$ on the magnetar surface to be $\sim 6 \times 10^{14} G$ [2], but it is observed that the magnetic field varies with patches to patches on the surface. Of course, we assume the spin period of a magnetar to be $\sim(5-8) s$, but we estimate all the calculations by taking the average value of spin period $\sim 7 \mathrm{~s}$. Near the magnetar surface at the local height scale, the potential difference is obtained by using the space charge limited flow (SCLF) model [20] that the part of the induced electric field will be left unshielded, and therefore a lower potential difference is obtained.

Zhu and Ruderman [27] found the Lorentz factor $\gamma \sim 5 \times$ $10^{6}$ when the primary particles approach the polar cap of Crab pulsar. In young Crab-like pulsars, the electron-positron pairs are produced by the curvature photons from the primary particles interacting with non-thermal synchrotron X-rays that produced in the same pairs. In pulsars inverse-Compton photons cannot screen the accelerating field; the primary particles continue to accelerate upto the Lorentz factor of $\sim 10^{7}$. Noted that Curvature Radiation is the dominant primary radiation mechanism and initiates the pair cascade. It is also noted that the primary source of high energy radiation in the polar cap model is curvature radiation. The very high energy electrons and positrons radiated from the polar cap along the open field lines produce curvature radiation.

In the polar gap region of a pulsar, Chi et al.[13] found that the relativistic electrons are restricted to flow along the strong magnetic field and they will radiate curvature photons with a typical energy $E_{c u r} \sim 6.4 \times 10^{8} \mathrm{P}^{1 / 2} \mathrm{eV}$.This value is found to be less by 3 orders than that of our results. In this paper, the photon energy radiated by the secondary electron-positron pairs via synchrotron radiation is observed larger than $E_{\text {syn }} \sim 2.6 \times 10^{8} B_{12} P\left(\frac{r}{3 R}\right)^{-3} \mathrm{eV}[13]$.

\section{Acknowledgement}

The authors would like to express thanks to Prof. H. L. Duorah, for his valuable help and fruitful discussions.

\section{References}

[1] Thompson, C. Lyutikov, M. and Kulkarni, S. R.: Astrophys. J., $574,322(2002)$

[2] Thompson, C. and Duncan, R. C.: MNRAS, 275, 255 (1995)
[3] Blaes, O., Blandford, R.,Goldreich, P. \&Madau, P.:ApJ, 343, 839-848 (1989)

[4] Cumming, A., Arras, P., \&Zweibel, E.: ApJ. 609, 999 (2004)

[5] Ruderman, M.A., and Sutherland, P.G.: Astrophys.J.196, 51 (1975)

[6] Cheng, K. S. \& Zhang, L.: Astrophys. J. 562, 918 (2001)

[7] Arons, J. \&Scharlemann, E. T.: Astrophys. J., 231, 854 (1979)

[8] Harding, A. K., \&Muslimov, A. G.: Astrophys. J., 508, 328 (1998)

[9] Cheng, K. S., Ho, C., \&Ruderman, M. A.:ApJ, 300, 500 (1986)

[10] Daugherty, J. K., \& Harding, A. K.:Astrophys. J. 458, 278 (1996)

[11] Zhang, B. \& Harding, A. K.:Astrophys. J. 535, L51-L54 (2000)

[12] Sturrock, P. A.:ApJ, 164, 529 (1971)

[13] Chi, X., Cheng, K. S. \& Young, E. C. M.:ApJ, 459, L83-L86 (1996)

[14] Zhang, L., \& Chang, K. S.:ApJ, 487, 370 (1997)

[15] Cheng, K. S., \& Zhang, L.:ApJ, 463, 271(1996)

[16] Reisenegger, A.: The International Workshop on Strong Magnetic Fields and Neutron Stars, La Habana, Cuba (arXiv: astro-ph/0307133v1)(2003)

[17] Goldreich, P.,\& Julian, W.H.:ApJ, 157, 869 (1969)

[18] Zhang, B. \& Harding, A. K.: Astrophys. J. 535, L51-L54 (2000)

[19] Thompson, C. and Duncan, R. C.: Astrophys. J. 473, 322 (1996)

[20] Muslimov, A. G., and Tsygan, A. I.: MNRAS, 255, 61(1992)

[21] Rudak, B. Dyks, J. \&Bulik, T.” High-energy radiation from pulsars: a challenge to polar-cap models, Prpc. of 270. WEHeraeus Seminar, "Neutron Stars, Pulsars and Supernova Remnants": astro-ph/0206101(2002)

[22] Arons: ApJ. 302, 301 ph/0206101 (1983)

[23] Halpern, J.P. and Ruderman, M.:ApJ, 415, 286 (1993)

[24] Erber, T.: Rev. Mod. Phys., 38, 626 (1966)

[25] Harding, A. K., Contopoulos, I., and Kazanas, D.:Astrophys.J. Vol. 525, No. 2, L125 (1999)

[26] Istomin, Ya, N. and Sobyanin, D. N.: Astronomy Letter, Vol. 33, No. 10, pp 660-672 (2007)

[27] Zhu, T. and Ruderman, M.: ApJ. 478, 701-712 (1997) 